\title{
AN OVERVIEW ON BASIC AND ADVANCED CHROMATOGRAPHIC TECHNIQUES
}

\section{Manisha Bhatti ${ }^{1}$, Ritika Puri ${ }^{2}$}

1,2 University Institute of Pharmaceutical Sciences, Chandigarh University, Gharuan, Mohali, Punjab, 140413

Article Info: Received 28 April 2019; Accepted 23 May. 2019

DOI: https://doi.org/10.32553/jbpr.v8i3.599

Address for Correspondence: Manisha Bhatti, University Institute of Pharmaceutical Sciences, Chandigarh University, Gharuan, Mohali, Punjab, 140413

Conflict of interest statement: No conflict of interest

\section{ABSTRACT:}

Chromatography is a technique for separating various inorganic and organic compounds. It is one of the separation techniques used as differential migration. It is more advantageous over conventional separating methods such as crystallization, solvent extraction and distillation. The purpose of review is to present various chromatographic techniques included a few advanced forms such as FC, HPLC,UPLC and UPCC (Super Critical chromatography).These are rapid forms of chromatographic techniques based on air pressure driven, optimized for rapid and precise separation of an organic compound.

Keywords: Chromatographic techniques, Column chromatography, HPLC, UPLC, FC, UPC ${ }^{2}$ etc

\section{INTRODUCTION}

Chromatography is an analytical method used for separation and analysis of various inorganic and organic compounds. It is a physical method based on differential migration pattern. In all types of chromatographic techniques the basic is that there is one mobile phase and one stationary phase. The moving phase runs through the static phase by picking up the components to be tested. At certain points in the stationary phase the different components of the sample/mixture are absorbed and thus separated from the mixture, and in this way the results may analyze. [1]

\section{Basic Principle:}

This technique depends on one of the phenomenon: adsorption and partition coefficient.
In adsorption there is a solid as static phase and a liquid or gas as a moving phase. Each solute particle which has to be separated has its own equilibrium distribution between adsorption onto the surface of the solid and solubility in the solvent, the least soluble or best adsorbed ones travel more slowly. This results a separation into bands containing different solute particles. [2, 3] in partition coefficient the static phase is a nonvolatile liquid which acts as a thin layer (or film) on the surface of an inert solid. The mixture of particles to be separated is carried by a gas or a liquid as mobile phase. The solute particles distribute themselves between the mobile and the stationary phase, and more soluble component in moving phase will reach at the end of the column first. [2, 3]

Table 1: Classification of Chromatographic Methods [4]

\begin{tabular}{|l|l|l|l|}
\hline Sr. No & Stationary Phase & $\begin{array}{l}\text { Mobile } \\
\text { Phase }\end{array}$ & Chromatographic Methods \\
\hline 1. & Solid & Liquid & Thin Layer Chromatography \\
\hline 2. & Solid & Liquid & Column Chromatography \\
\hline 3. & Solid & Liquid & High Pressure Liquid Chromatography \\
\hline 4. & Solid/Liquid & Gas & Gas Chromatography \\
\hline
\end{tabular}


Manisha Bhatti et al., Journal of Biomedical and Pharmaceutical Research

\begin{tabular}{|l|l|l|l|}
\hline 5. & Solid & Liquid & Affinity Chromatography \\
\hline 6. & Solid & Liquid & Gel Permeation Chromatography \\
\hline 7. & Solid & Liquid & Ion Exchange Chromatography \\
\hline 8. & Solid & Liquid & Ultra performance Liquid Chromatography \\
\hline 9. & Solid & Liquid & Flash Chromatography \\
\hline 10. & Solid & Liquid & Ultra Performance Convergence Chromatography \\
\hline
\end{tabular}

\section{Thin Layer Chromatography (TLC)}

In TLC an absorbent material which acts as a stationary phase is spread over the plane flat glass or plastic plates. The adsorbents such as alumina or silica spread on an inert base made of glass, aluminum foil or insoluble plastics in TLC. The particles to be separated are spotted with the help of capillary at the bottom of TLC plate and allowed to dry for few minutes. Then the prepared plate is placed in a closed chamber containing mobile phase in such a way so that the level of liquid is below the spot. The solvent ascends the plate by capillary action. The plate is removed from chamber when the solvent front approaches $3 / 4$ thof the plate and the position of the solvent front recorded before it is dried that is this allow the $\operatorname{Rf}$ value to be calculated. $[2,3,5,6]$

$\mathrm{Rf}=$ Distance Travelled by solute particle

Distance Travelled by solvent

$\mathrm{Rf}=$ Retention Factor

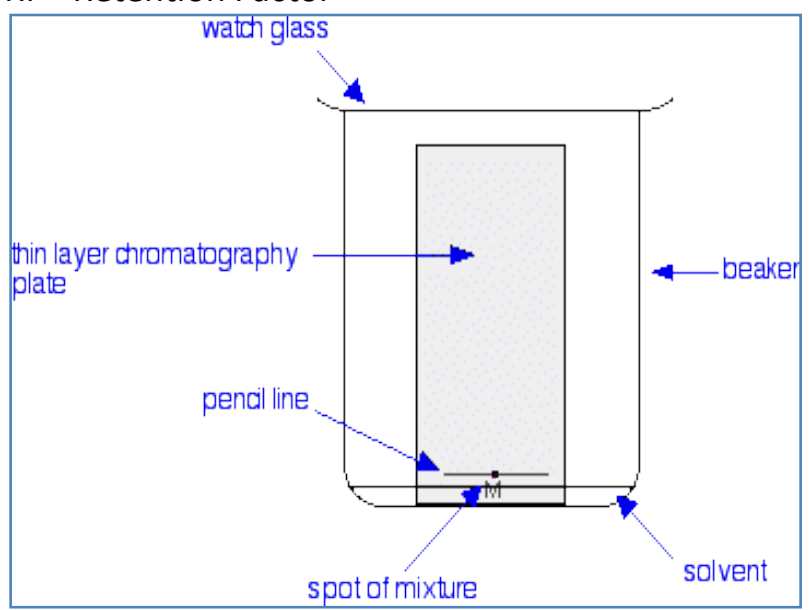

Figure 1: Technique of TLC [4]

\section{Column Chromatography}

It is a preparative technique used to purify compounds depending upon their polarity or hydrophobicity. In this technique a mixture of molecules is separated based on their differential partitioning between a mobile phase and a stationary phase.Under this methods the columnis used made of glass tube with a diameter about 5 $\mathrm{mm}$ to $50 \mathrm{~mm}$ and a height of $5 \mathrm{~cm}$ to $1 \mathrm{~m}$ with a tap and some kind of a filter such as a glass frit or glass wool plug to prevent the loss of the stationary phase at the bottom. Generally a column can be prepared by the dry method, and the wet method. The technique typically requires mesh $70-230$ (63 $-200 \mu \mathrm{m}$ ) silica gel. The main advantage of CC is the relatively low cost and disposability of the stationary phase used in the process. The advanced form of CC is called high performance/high pressure/ high-resolution and high-speed liquid chromatography. $[6,7]$

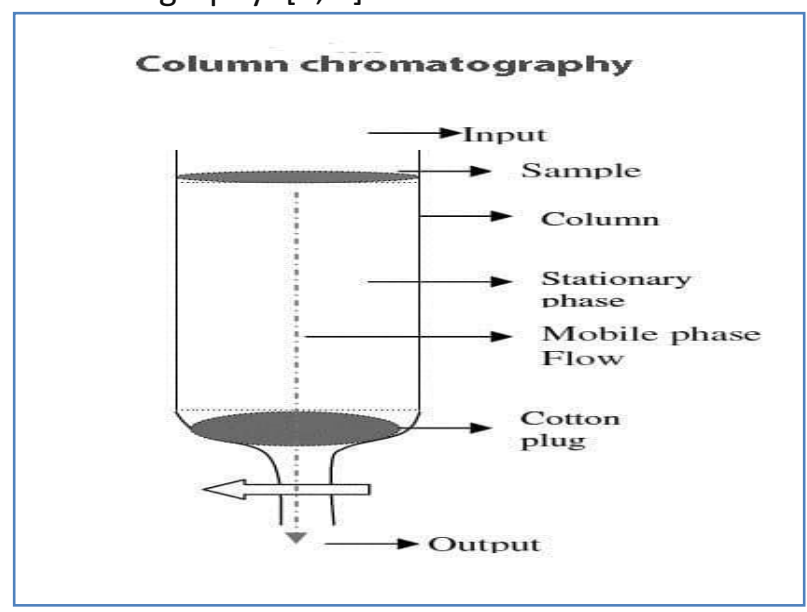

Figure 2: Technique of Column Chromatography

[7]

\section{High Performance/pressure Liquid Chromatography (HPLC)}

It is a technique in analytical world used for separation, identification and quantification of each component in a mixture. Separation of components relies on the pump to pass a pressurized liquid solvent through a column filled with a solid adsorbent material. The components get separated on the basis of their polarity and adsorption rates[8]A schematic representation of HPLC consist of solvent reservoirs, solvent degasser,gradient valve, mixing vessel, high pressure pump, switching valve in injection position and loading position, sample injection 
loop, guard column, analytical column, detectors and waste collector. $[7,8,9,10]$

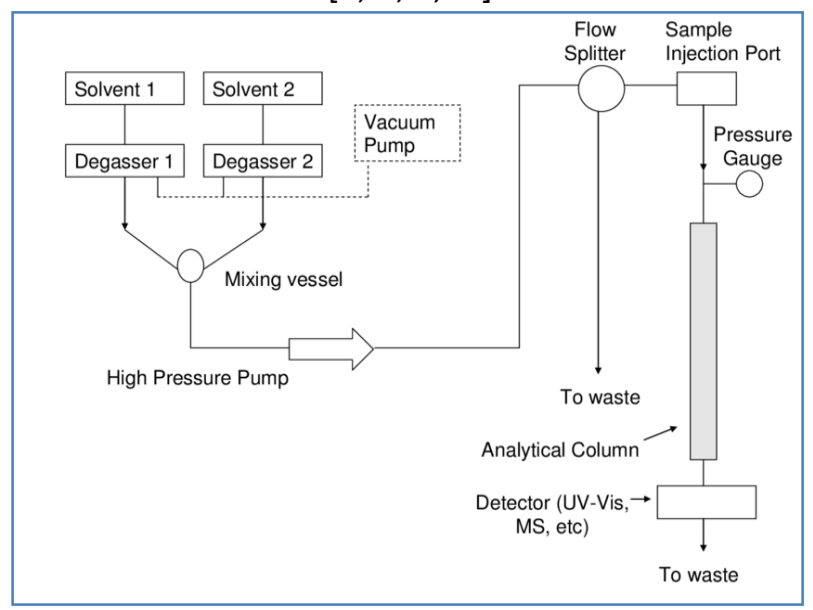

Figure 3: Instrumentation of HPLC [9]

\section{Gas Chromatography (GC)}

This is the one of the chromatographic techniques used for separation and analysis of compounds in vapor form without their decomposition. Under this, the moving phase is a carrier gas, and the static phase is a fine layer of liquid or polymer on an inert solid support. The inert gasesor uncreative gases are mostly used as carrier gas. Usually helium, neon and nitrogen are used. The gaseous or vaporized particles being analyzed by interaction with the walls of the column coated with a stationary phase. Under the influence of carrier gas every compound which is to be separated will elute at a different speed and time. $[10,11]$

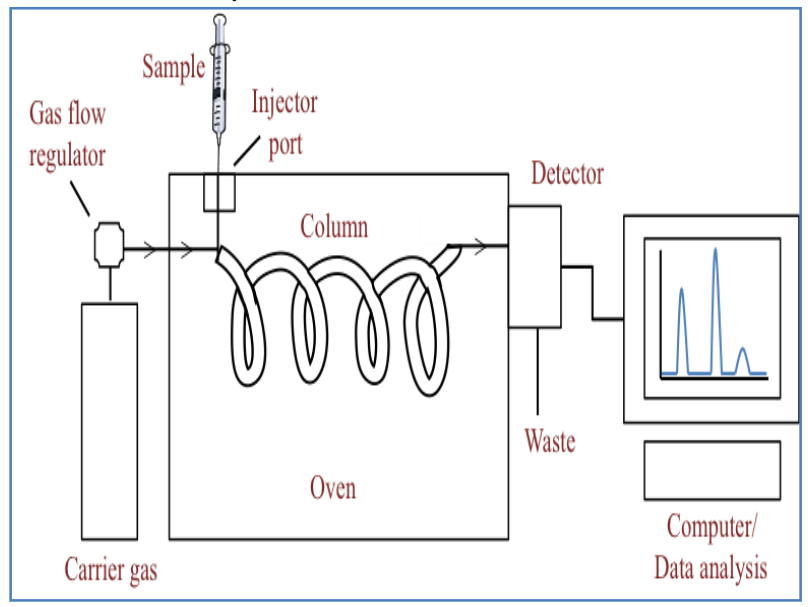

Figure 4: Instrumentation of Gas Chromatography [11]

\section{Affinity Chromatography}

This technique is particularly used for the separation and purification of biochemicals such as enzymes, hormones, antibodies, nucleic acids, and specific proteins [12]. It exploits the differences in interaction strengths between the different biomolecules within a mobile phase and a static phase. Under this stationary phase is a gel matrix of any polymer such as agarose and a mobile phase may be any liquid, in few cases buffer solution also act as mobile phase. Components get separated by making a complex with the solid matrix, and retained in the column, while free protein particles will leave the column first. $[13,14]$

\section{Gel- Permeation or Molecular Sieve Chromatography}

In this techniquemolecular size is prime factor of separation.Various macromolecules separated with method. This technique isalso helpful to determine the molecular weights macromolecules. [15]The stationary phase consists of inert molecules havingfine pores.Dextran, agarose, Sephadek $G$ and polyacrylamide are the mostly used for column packing materialand the mobile phase is the solution containing molecules of different dimensions. The moleculeswhich have larger sizes than pores of stationary phasecannot permeate into gel particles, and pass through spaces between porous particles, and move rapidly from the column. Whereas molecules of relatively smallersizes diffused into pores, and ultimately leave the column later. [16]

\section{Ion- Exchange Chromatography}

Under this ions and polar molecules based on their affinity get separated. This process used for charged molecule such asamino acids, proteinsand nucleotides.Separation is based on electrostatic interactions among charged protein particles, and solid matrix of stationary phase. Static phase is prepared with ionic matrix having opposite charge to that of the protein or amino acid particle is to be separated, and the interaction of the component to be separated and the column is achieved with ionic terms. Separation occurs either by changing $\mathrm{pH}$, or ionic strength of the buffer solution. $[17,18,19]$

\section{Ultra Performance Liquid Chromatography (UPLC):}

This is an advanced technique in liquid chromatography, which significantly reduces the separation time and solvent consumption. It takes about nine fold decrease in analysis time as 
compared to the conventional HPLC because of its less than $2 \mu \mathrm{m}$ in particle size column packing. By reduction in separation times without reducing the quality of the separation is vital analytical information. The smaller will be the particle size of component to be separated higher will be the efficiency of column. [20-25]

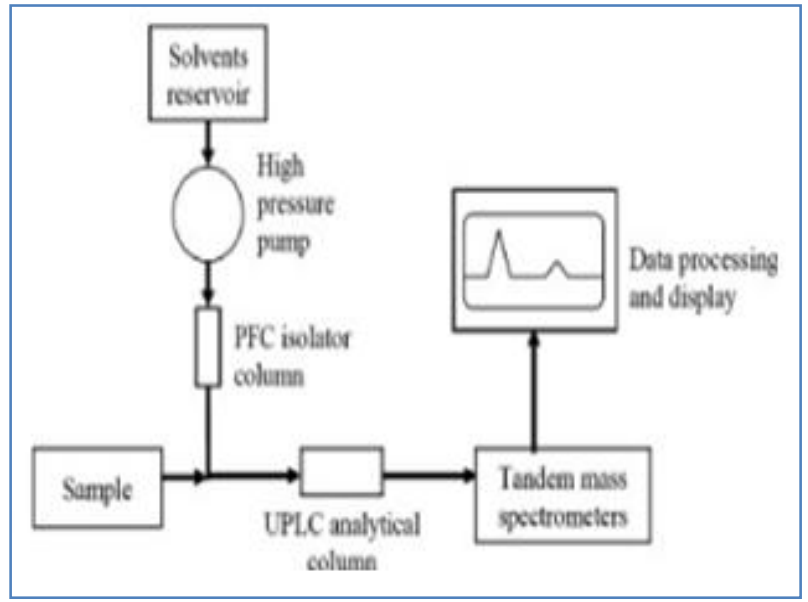

Figure 5: Instrumentation of UPLC [25]

\section{Flash chromatography (FC):}

Conventional Column chromatography technique is often time consuming. Whereas the flash can speed up the flow rate of the column and made it a fast separating process.In this a pressure of about 10 psi of air or nitrogen use to force and run the moving phase into the column. Under this influence the rate of the moving phase is increased. Moreover, with a finer grade (mesh 230 $-400(40-63 \mu \mathrm{m})$ of alumina or silica is used as stationary phase in flash chromatography which helps to increase the speed of the separation. [26, 27]

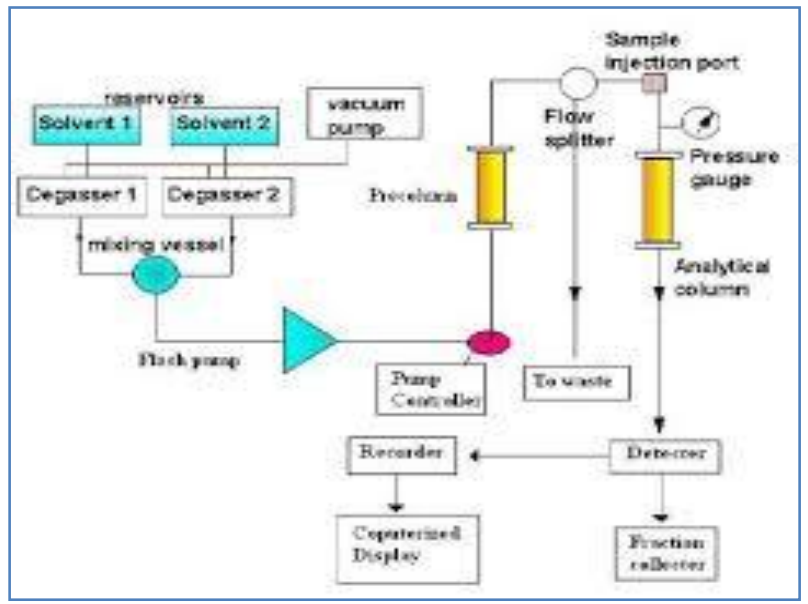

Figure 6: Instrumentation of Flash chromatography [27]
Ultra Performance Convergence Chromatography $\left(\mathrm{UPC}^{2}\right)$

It is the fastest, most reliable and robust technologies available for chromatographic testing. It is novel method for tackling hydrophobic components such as chiral compounds, lipids, thermo labile substances and polymers. This method is a variant of supercritical fluid chromatography and Supercritical fluid chromatography is one of the most important column chromatography methods. [10,22,23,24] UPC $^{2}$ is a technique for analysis of non-volatile and semi-volatile extractable, as well as polar and nonpolar compounds.[10,24]

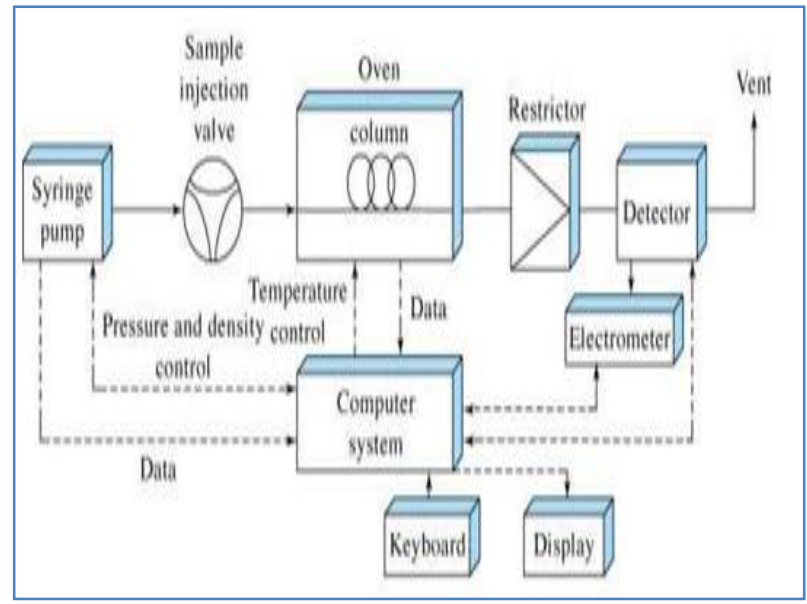

Figure 7: Instrumentation of Flash chromatography [10]

\section{CONCLUSION}

Chromatographic testing has become vital in industries and laboratories for separation, purification and quantification. In term of scientific advances, chromatography is considered to be the one of the major innovations in the past few years. Upgraded forms of many chromatography techniques increase productivity reliability and robustness.

\section{REFERENCE}

1. Kaushal C, Srivastava B. A process of method development: A chromatographic approach. J Chem Pharm Res 2010; 2(2): 519- 545.

2. Sherman J, Fried B, Dekker M. New York, NY: Handbook of Thin-Layer Chromatography; 1991.

3. Donald PL, Lampman GM, Kritz GS, Randall G. Engel introduction to organic laboratory 
Manisha Bhatti et al., Journal of Biomedical and Pharmaceutical Research

techniques.4th ed. Thomson Brooks/Cole; 2006. pp. 797-817.

4. Heftmann E (ed) Chromatography, Fundamentals and applications of chromatography and related differential migration methods. Part A: fundamentals and techniques. Part B: applications. J Chromatogr Library Ser vols 69A and 69B. Elsevier, Amsterdam 2004; 6th edn.

5. Kumar S, Jyotirmayee K, Sarangi M. Thin Layer Chromatography: A Tool of Biotechnology for Isolation of Bioactive Compounds from Medicinal Plants. Int. J. Pharm. Sci. Rev. Res. 2013; 18(1), 126-132

6. Chromatography, available on: https:// satyapsingh. files.wordpress.com/2012/09 /chromatography-anddistillation.pdf

7. Das M, Dasgupta D. Pseudo-affinity column chromatography based rapid purification procedure for T7 RNA polymerase. Prep Biochem Biotechnol. 1998;28:339-48.

8. Reynolds $\mathrm{DW}$, Facchine $\mathrm{KL}$, Mullaney JF, Alsante KM, Hatajik TD, Mott MG. Available Guidance and Best Practices for Conducting Forced Degradation Studies. Pharmaceutical Technology, 2002; 48-56.

9. Gentili A, Caretti F, Ascenzo GD. Simultaneous determination of water-soluble vitamins in selected food matrices by liquid chromatography/electrospray ionization and mass spectrometry, Rapid Communications in Mass Spectrometry. 2008. p. 2029- 2043.

10. Kondeti R. R, Mulpuri K. S, Meruga B. Advancements in column chromatography: $A$ review World Journal of Pharmaceutical Sciences.2014;2 (9) 1375-1383

11. Chaturvedi. K.K, Nanda R.K. Review on Hyphenated Gas Chromatography. International Journal of Pharmaceutical Sciences Research and Review.2010; 5(3),1827

12. Wilchek $M$, Chaiken I. An overview of affinity chromatography in affinity chromatographyMethods and protocols. Humana Press. 2000:1-6.

13. Firer MA. Efficient elution of functional proteins in affinity chromatography. J Biochem Biophys Methods. 2001;49:433-42.
14. Walls D, Loughran ST. Protein chromatography: Methods and protocols, methods in molecular biology. 2011;681

15. Helmut D. Gel Chromatography, gel filtration, gel permeation, molecular sieves:a laboratory hand book. Springer-Verlag; 1969.

16. Determann H. Gel chromatography gel filtration, gel permeation, molecular sieves:a laboratory handbook. Chapter 2. Materials and Methods. 2012.

17. Karlsson E, Ryden L, Brewer J Protein purification. Principles, High Resolution Methods, and Applications. Ion exchange chromatography. 2nd ed. New York: Wiley; 1998.

18. Amercham Biosciences. Ion Exchange chromatohgraphy, Principles and methods, Amercham Pharmacia. Biotech SE. 2002;751.

19. An Encyclopedia of Chemical, Drugs and Biologicals, 13th ed., Merck Research Laboratories. Division of Merck \& Co Inc. Whitehouse Station, NJ. The Merck index. 2001. p. 245.

20. Jeffery GH and Basselt J. Vogel's Text Book of Quantitative chemical analysis. 6th ed. Pearson education pvt. Ltd. Singapore; 2003. P. 21-23.

21. Liu $Y$, Lee ML. Ultra high pressure liquid chromatography using elevated temperature. Journal of Chromatography. 2006; 1104 (12):198-202.

22. Patel RM et al. Stability Indicating HPLC Method Development- A Review. Int Res J Pharmacy 2011; 2(5): 79-87.

23. Novakova L, Matysova L, Solich P. Advantages of application of UPLC in pharmaceutical analysis. Talanta: 2006. P. 908-918.

24. Taleuzzaman M, Ali S, Gilani SJ, Imam SS and Hafeez A. Ultra Performance Liquid Chromatography (UPLC): A Review. Austin J Anal Pharm Chem. 2015; 2(6)1056.

25. Thammana M. A review on high performance liquid chromatography. RRJPA. 2016; 5(2): 2228.

26. Swathi G, Srividya A, Ajitha A and Rao V.U.M. Review on: Flash Chromatography. WJPPS. 2015; 4(8),281-296

27. G. swati, Kandlakoya, Flash chromatography, Aug 2015, Available on: www.wjpps.com /download/article/1438354107.pdf 